\title{
Connecting the Interstellar Gas and Dust Properties in Distant Galaxies Using Quasar Absorption Systems
}

\author{
Monique C. Aller ${ }^{1,2}$, Varsha P. Kulkarni ${ }^{2}$, Donald G. York ${ }^{3}$, Daniel E. \\ Welty $^{3}$, Giovanni Vladilo ${ }^{4}$, Debopam Som ${ }^{2}$, Kyle Lackey ${ }^{2}$, Eli Dwek ${ }^{5}$, \\ Nassim Beiranvand $^{2}$ and Sean Morrison ${ }^{2}$ \\ ${ }^{1}$ Dept. of Physics, Georgia Southern University, Statesboro, GA, 30460, USA \\ email: maller@georgiasouthern.edu \\ ${ }^{2}$ Dept. of Physics \& Astronomy, University of South Carolina, Columbia, SC, 29208, USA \\ ${ }^{3}$ Dept. of Astronomy \& Astrophysics, University of Chicago, Chicago, IL, 60637, USA \\ ${ }^{4}$ Osservatorio Astronomico di Trieste, Via Tiepolo 11, I-34143 Trieste, Italy \\ ${ }^{5}$ NASA GSFC, Greenbelt, MD, 20771, USA
}

\begin{abstract}
Gas and dust grains are fundamental components of the interstellar medium and significantly impact many of the physical processes driving galaxy evolution, such as star-formation, and the heating, cooling, and ionization of the interstellar material. Quasar absorption systems (QASs), which trace intervening galaxies along the sightlines to luminous quasars, provide a valuable tool to directly study the properties of the interstellar gas and dust in distant, normal galaxies. We have established the presence of silicate dust grains in at least some gas-rich QASs, and find that they exist at higher optical depths than expected for diffuse gas in the Milky Way. Differences in the absorption feature shapes additionally suggest variations in the silicate dust grain properties, such as in the level of grain crystallinity, from system-to-system. We present results from a study of the gas and dust properties of QASs with adequate archival IR data to probe the silicate dust grain properties. We discuss our measurements of the strengths of the 10 and $18 \mu \mathrm{m}$ silicate dust absorption features in the QASs, and constraints on the grain properties (e.g., composition, shape, crystallinity) based on fitted silicate profile templates. We investigate correlations between silicate dust abundance, reddening, and gas metallicity, which will yield valuable insights into the history of star formation and chemical enrichment in galaxies.
\end{abstract}

Keywords. (galaxies:) intergalactic medium, (galaxies:) quasars: absorption lines, galaxies: ISM, (ISM:) dust, extinction, ISM: abundances

\section{Silicate Dust in Gas-Rich QASs}

QSO absorption systems (QASs) probe the gas and dust properties in galaxies along the sightlines to background quasars. Evidence for dust in gas-rich QASs includes refractory element depletions and background quasar reddening. In the Milky Way 66\% of the core mass of interstellar dust is found in silicate grains (e.g., Zubko et al. 2004). Our finding of $9.7 \mu \mathrm{m}$ silicate dust absorption in five $0.4 \leqslant z \leqslant 1.4$ dusty QASs (Kulkarni et al. 2011), using the Spitzer Infrared Spectrograph (IRS), led us to obtain IRS spectra covering the $9.7 \mu \mathrm{m}$ (and for some $18 \mu \mathrm{m}$ ) absorption feature(s) for 8 more dusty, gas-rich $0.1 \leqslant \mathrm{z} \leqslant 1.4$ QASs. We use these data in combination with archival optical spectra covering gas-phase metal lines to explore the correlations between gas and dust properties in QASs.

We find differences in the $10 \mu \mathrm{m}$ silicate dust absorption feature substructure between QASs, which suggests dust grain property variations. This is illustrated by comparing the absorption spectrum for the $\mathrm{z}=0.886$ QAS toward PKS 1830-211 with that for the 

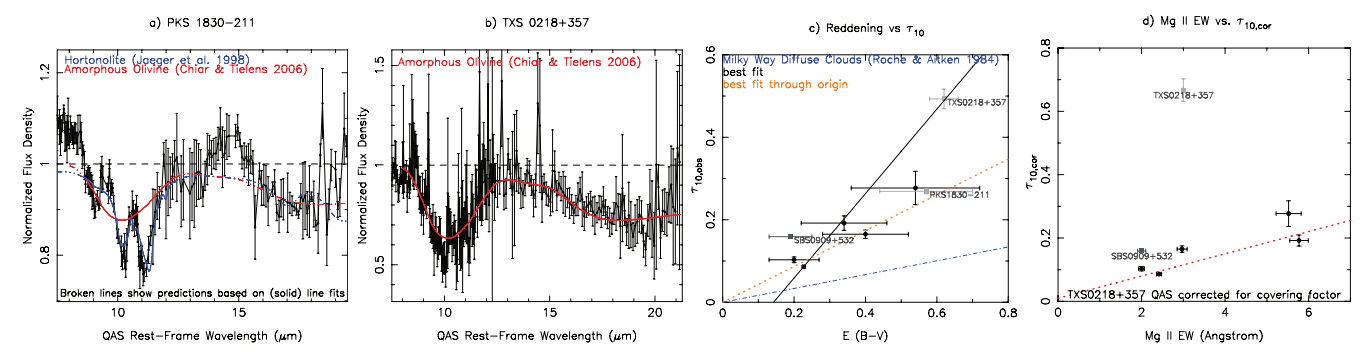

Figure 1. Template profile fits to silicate dust absorption features for (a) the $z=0.886$ QAS toward PKS 1830-211 and (b) the $\mathrm{z}=0.685$ QAS toward TXS 0218+357 illustrating the range in spectral substructure. Adapted from Aller et al. 2012; Aller et al. 2014. (c) Possible correlation between the measured $\tau_{10}$ and the reddening, $\mathrm{E}(\mathrm{B}-\mathrm{V})$. If corrected for the covering factor, the lensed QASs (labeled squares) will be further offset from the diffuse Milky Way clouds relationship (see Aller et al. 2014). (d) Suggestion of a trend between the Mg II $\lambda 2796$ rest-frame EW and $\tau_{10}$. Values in $\mathrm{c}-\mathrm{d}$ for the $\mathrm{z}=0.83$ QAS toward SBS $0909+532$ are preliminary.

$\mathrm{z}=0.685$ QAS toward TXS $0218+357$ (Fig. 1, a-b). In both lensed systems the QAS is hosted by a molecule-rich (e.g., $\mathrm{CO}, \mathrm{HCO}+, \mathrm{H}_{2} \mathrm{O}$, and $\mathrm{NH}_{3}$ ) face-on spiral galaxy. While the TXS 0218+357 QAS $10 \mu \mathrm{m}$ absorption feature is well-fit by an amorphous olivine template profile, this profile does not well-reproduce the peak wavelength, breadth, and substructure of the $10 \mu \mathrm{m}$ feature in the PKS 1830-211 QAS. Profile fits indicate that the $10 \mu \mathrm{m}$ absorption feature properties can instead be reproduced by crystalline olivine $\left(\mathrm{Mg}_{2 x} \mathrm{Fe}_{2-2 x} \mathrm{SiO}_{4}\right)$ silicate templates, suggesting that the ISM silicate dust in this QAS may be more crystalline than that in the predominately amorphous Galactic ISM.

\section{Silicate Dust Trends and Correlations}

We investigate correlations between the $10 \mu \mathrm{m}$ peak optical depth $\left(\tau_{10}\right)$ and both the reddening and the $\mathrm{Mg}$ II $\lambda 2796$ rest-frame equivalent width $(\mathrm{EW})$. The $\tau_{10}$-reddening correlation is 3-6 times steeper than that found for Milky Way diffuse ISM clouds (Fig. 1, c). This may suggest differences in the silicate grains (e.g., larger grains yielding a lower UV extinction) or stellar population differences (e.g., a higher fraction of O-rich stars could increase the amount of silicate dust). We find a weak trend between the $\tau_{10}$ and the Mg II $\lambda 2796 \mathrm{EW}$ (Fig. 1, d). Since the Mg II line is saturated in most systems, it may be a proxy for the velocity spread, in which case the suggested trend may indicate that silicate-dust-rich QASs are on average more massive or have stronger outflows.

This work uses observations made by the Spitzer Space Telescope, and was supported by NASA through ADAP grant NNX14AG74G and by an award issued by JPL/Caltech, and from US-NSF grants AST-0908890 and AST-1108830 to the U. of S. Carolina.

\section{References}

Aller, M.C., Kulkarni, V.P., York, D.G., Vladilo, G., Welty, D.E., \& Som, D. 2012, ApJ, 748, 19 Aller, M.C., Kulkarni, V.P., York, D.G., Welty, D., Vladilo, G., \& Liger, N. 2014, ApJ, 785, 36 Chiar, J.E., \& Tielens, A.G.G.M. 2006, ApJ, 637, 774

Jäger, C., Molster, F.J., Dorschner, J., Henning, Th., Mutschke, H., \& Waters, L.B.F.M. 1998, $A \mathscr{\mho} A, 339,904$

Kulkarni, V.P., Torres-Garcia, L.M., Som, D., York, D.G., Welty, D.E., \& Vladilo, G. 2011, ApJ, 726,14

Roche, P.F., \& Aitken, D. K. 1984, MNRAS, 208, 481

Zubko, V., Dwek, E., \& Arendt, R.G. 2004, ApJS, 152, 211 